\title{
Strength asymmetry of the knee extensors and physical activity in middle-aged women
}

\author{
Michal Lehnert*, František Chmelík, Roman Cuberek, and Vendula Svobodová \\ Faculty of Physical Culture, Palacký University, Olomouc, Czech Republic
}

Copyright: (c) 2014 M. Lehnert et al. This is an open access article licensed under the Creative Commons Attribution License (http://creativecommons.org/licenses/by/4.0/).

Background: Differences between muscle strength and power of lower limbs is one of the factors associated with falls in the elderly population. Muscle strength asymmetry of lower limbs increases with age. Objective: The aim of the study is to assess differences in the volume and intensity of physical activity (PA) in the subgroups of women being at higher and lower risk of falls (with and without strength asymmetry of the knee extensors of dominant and non-dominant lower limb). Methods: Data from 42 women (age $56.3 \pm 4.4$ years; weight $76.5 \pm 16.1 \mathrm{~kg}$; body height $164.3 \pm 5.2 \mathrm{~cm}$; body mass index $28.1 \pm 5.3 \mathrm{~kg} \cdot \mathrm{m}^{-2}$ ) were used for the purpose of the presented analysis. Furthermore the sample was divided into subgroups with lower and higher strength asymmetry of quadriceps muscle (as a criterion the asymmetry greater than $15 \%$ was chosen). Absolute concentric peak torque of the knee extensors was evaluated by an isokinetic dynamometer IsoMed 2000 in a sitting position at angular velocity of $180^{\circ} \cdot \mathrm{s}^{-1}$. PA was monitored using Yamax SW-700 pedometers throughout 7 consecutive days and using the Czech version of standardized International Physical Activity Questionnaire. Results: Statistically significant differences between groups with lower and higher strength asymmetry were found in the amount of self-reported vigorous PA $(p=.04 ; d=0.6)$ only. Differences between the observed groups were not significant in self-reported moderate PA and walking. There were also no significant differences in the daily numbers of steps measured objectively. Conclusions: The results of the study indicate that from the point of view of strength asymmetry of knee extensors as a factor associated with falls in middle-aged women, intensity of PA could be an important characteristic of PA. This suggestion should be taken into account in habitual PA and training programs in middle-aged population.

Keywords: bilateral differences, sedentary population, peak torque, quadriceps

\section{Introduction}

Decrease of muscle strength with age in men and women has been sufficiently documented in literature (Borges, 1989; Keller \& Engelhardt, 2013; Perry, Carville, Smith, Rutherford, \& Newham, 2007). Results of studies (Lindle et al., 1997; Stoll, Hubert, Seifert, Michel, \& Stucki, 2000) confirmed that this decrease accelerates after the age of 50 . For people over 50 , the decrease in muscle strength is by $12-15 \%$ per decade (Hughes et al., 2001). The annual decline is by $1.5 \%$ between ages 50 and 60 and by $3 \%$ thereafter, which is primarily connected with a loss of muscle mass by 1-2\% per year (Stoll et al., 2000; Von Haehling, Morley, $\&$ Anker, 2010). The loss of muscle mass occurs due to reduction in the number of muscle fibers, muscle

\footnotetext{
* Address for correspondence: Michal Lehnert, Department of Sport, Faculty of Physical Culture, Palacký University, tř. Míru 115, 77111 Olomouc, Czech Republic. E-mail: michal.lehnert@upol.cz
}

fiber size and denervation of motor units (Macaluso \& De Vito, 2004; Von Haehling et al., 2010). The above mentioned changes are associated with decreased postural stability and increased risk of falls in everyday activities (Ikezoe, Asakawa, Shima, \& Ichihashi, 2009; Ochi et al., 2010; Semaltianoy, Kabitsis, Harahousou, \& Lazou, 2011).

It was confirmed that the cross sectional area of the quadriceps is increasingly associated with aging than other thigh muscles (Frontera et al., 2000; Ochi et al., 2010) and muscles of upper body (Aniansson, Hedberg, Henning, \& Grimby, 1986). Strength of the quadriceps is important to carry out daily activities, as it ensures the stability of the knee joint, which is needed for upright posture and gait. Quadriceps controls the movements while walking down the stairs, up the stairs, sitting down and when squatting (Kapandji, 2002; Véle, 2006). Thus, muscle strength and power of quadriceps are considered to be predictors of falls in elderly (Semaltianoy et al., 2011; Skelton, Kennedy, 
\& Rutherford, 2002). It has also been confirmed that muscle use during regular physical activity (PA) can influence positively muscle mass, muscle composition, muscle strength and other characteristics in older persons and that older men and women who have active lifestyle have a lower risk of falls and incident mobility limitation (Daly et al., 2008; Gauchard, Tessier, Jeandel, \& Perrin, 2003; King, Rejeski, \& Buchner, 1998). This fact has fundamental consequences, because in the elderly the more frequent occurrence of falls often results in loss of functional independence (Stokes \& Linsay, 1996).

However, it was also suggested that other risk factors for falls could be the difference between strength and power of muscles of the knee and ankle, including knee extensors (Perry et al., 2007; Skelton et al., 2002) and that considerable strength difference of the muscle of lower limbs, including knee extensors compromises mobility and balance in healthy elderly women (Portegijs, 2008). Strength asymmetry or bilateral percentage difference between muscle strength of knee extensors may be a result of leg dominance (Lanshammar \& Ribom, 2011) or unilateral pathology (Suetta et al., 2007). In subjects with the presence of muscle asymmetry slower walking was found, which demonstrates the relationship between asymmetry and mobility (LaRoche, Cook, \& Mackala, 2012) and association between asymmetrical walking patterns and a risk of falls was also confirmed (Sadeghi, Allard, Prince, \& Labelle, 2000; Yogev, Plotnik, Peretz, Giladi, \& Hausdorff, 2007). It has been proven that strength asymmetry increases with age (Carabello, Reid, Clark, Philips, \& Fielding 2010; Perry et al., 2007). However how the occurrence of quadriceps asymmetry relates to habitual PA in the middle-aged is not clear. Moreover, in the studies focused on the relationship between strength, PA, injury risk and aging, people older than 65 years were mostly observed.

The aim of the study is to assess differences in volume and intensity of PA in the subgroups of women over 50 years old being at higher and lower risk of falls (with and without strength asymmetry of the knee extensors of dominant and nondominant lower limb).

\section{Methods}

\section{Subjects}

Data from initial measurements within the frame of study "Analysis of health-related benefits of walking: Assessment of walking intervention in sedentary adults" were used for the purpose of this study. One hundred and twenty seven healthy women with sedentary occupations participated in the study. All women were fully informed about the aim of the study and the procedures employed in the study. Written informed consent agreeing to the measuring procedures and the use of the data for further research was obtained. Out of this number, data from 42 women (age $56.3 \pm 4.4$ years; weight $76.5 \pm 16.1 \mathrm{~kg}$; height $164.3 \pm 5.2 \mathrm{~cm}$; body mass index $28.1 \pm 5.3 \mathrm{~kg} \cdot \mathrm{m}^{-2}$ $[\mathrm{M} \pm \mathrm{SD}]$ ) were used for the purpose of this analysis. Reasons for exclusion were orthopaedic disorder of the lower extremities and trunk, previous surgery of a lower extremity, unilateral or bilateral knee pain, severe osteoporosis, hypertension, and missing data from one of the legs from the above mentioned reasons, and unsatisfactorily executed movement task during testing because of insufficient familiarisation or due to other reasons. Leg dominance was verified (preferred kicking leg) before testing. To divide our sample into subgroups with lower $(n=32$; age $56.5 \pm 4.5$ years; body weight $78.0 \pm 16.7 \mathrm{~kg}$; body height $164.9 \pm 4.6 \mathrm{~cm}$; body mass index $\left.28.5 \pm 5.6 \mathrm{~kg} \cdot \mathrm{m}^{-2}[\mathrm{M} \pm \mathrm{SD}]\right)$ and higher $(n=10$; age $56.0 \pm 4.2$ years; body weight $71.7 \pm 13.4 \mathrm{~kg}$; body height $162.5 \pm 6.6 \mathrm{~cm}$; body mass index $\left.27.1 \pm 4.2 \mathrm{~kg} \cdot \mathrm{m}^{-2}[\mathrm{M} \pm \mathrm{SD}]\right)$ strength asymmetry, respectively with higher and lower risk of falls, as a criterion we chose the asymmetry of quadriceps muscle strength greater than 15\% (Davies, Heiderscheit, \& Brinks, 2000). We also took into account the results of studies in the elderly, in which an average 14\% (Perry et al., 2007) or less difference between the power of the muscles of the legs was found and also the results of other studies in the elderly (Hunter, Thompson, \& Adams, 2000), in which the side to side differences in strength were smaller.

\section{Procedures}

Bilateral isokinetic strength of the knee extensors was measured using an isokinetic dynamometer IsoMed 2000 (D. \& R. Ferstl GmbH, Hemau, Germany). Prior to testing the participants completed non-specific warm-up exercises, which included cycling on a stationary bicycle ergometer for 6 minutes at a self-regulated low to moderate intensity, stretching exercises which targeted the main muscle groups involved during testing, and 10 half-squats/squats with increasing range of movement. The warm-up routine was performed under the supervision of the researcher. At the end of the warm-up women performed imitated eccentric and concentric actions of quadriceps against the resistance created by the hand of researcher to become familiar with following movement task.

Women were tested in a sitting position with a hip angle of $100^{\circ}$. For fixation of the pelvis and the thigh of the tested leg, fixed straps were used; the shoulders were fixed by shoulder pads in the ventral-dorsal and 
cranial-caudal direction. The axis of rotation of the dynamometer was aligned with the axis of rotation of the knee (lateral femoral epicondyle). The arm of the dynamometer lever was fixed to the distal part of the shin and the lower edge of the shin pad was placed $2.5 \mathrm{~cm}$ over the medial apex malleolus. Individual seat settings were stored on the computer before measuring the right leg and were automatically activated in the process of measuring the left leg and follow-up testing respectively. At the beginning of the follow-up testing, individual settings were rechecked and adjusted if necessary. The participants were instructed to hold the handgrips located at the side of the seat during all testing efforts. An angular velocity of $180^{\circ} \cdot \mathrm{s}^{-1}$ was used for the measurement. Static gravitational correction was applied according to the manufacturer's procedures. The testing range of motion was $80^{\circ}$ and was set from $10-90^{\circ}$ of knee flexion (with $0^{\circ}=$ full voluntary extension). The testing protocol consisted of two sets (warm-up and testing). In the first warmup set women performed five eccentric/concentric reciprocal actions with flexion movements performed first) with a progressive rise in the muscle action until a maximum action was performed. After a 2 minute rest women performed a set of three maximum repetitions. Because of the problems with execution of eccentric actions these results were not used for further analysis in this study. The rest time between the measurement of the dominant leg (DL) and non-dominant leg (NL) was 3 minutes, and the right leg was measured first. During the testing procedure the participants were provided with concurrent visual feedback in the form of an isokinetic strength curve displayed on the dynamometer monitor. Verbal encouragement was provided. Absolute concentric extension peak torque (PT) was used to assess strength of the knee extensors.

PA of the participants was monitored both objectively and subjectively. Yamax SW-700 pedometers (Yamax Co., Yasama Corp., Tokyo, Japan) which are considered to be valid tools for research purposes (Tudor-Locke, Bassett, Shipe, \& McClain, 2011) were used to measure the daily numbers of steps on 7 consecutive days. The participants were instructed on how to use the pedometers a day prior to the start of the monitoring. They wore the pedometers at the hip bone on the right side of their waist from the morning until the evening and took them off for swimming, personal hygiene, and sleeping. The participants were instructed to reset the pedometer each morning and to record their number of achieved steps into their personal $\log$ each evening.

Subjective data on weekly PA were obtained using the Czech version of standardized (Craig et al., 2003) International Physical Activity Questionnaire (IPAQ).
Translate and back-translate procedure for the Czech version of the questionnaire was performed as suggested (IPAQ group, 2014a). Participants completed the questionnaires online via the Indares.com system. Data from the IPAQ questionnaire were processed according to "Guidelines for data processing and analysis of the international physical activity questionnaire" (IPAQ group, 2014b).

\section{Statistical analysis}

Descriptive statistics and Mann-Whitney $U$ test to compare the differences between participants at higher and lower risk of falls were computed using IBM SPSS Statistics 22. Statistical significance was considered at the level of $p=.05$. Coefficient $d$ (Cohen, 1988) was used to evaluate the effect size.

\section{Results}

Medians, interquartile ranges, statistical significance of Mann-Whitney $U$ test, and Cohen's $d$ for all measurements are shown in Table 1. There were no significant differences between the groups of women with concentric extension PT asymmetry in the objectively measured daily number of steps. Self-reported data from the IPAQ questionnaire revealed statistically significant difference in the amount of vigorous PA between the observed groups of women. The differences in the amount of self-reported PA of moderate intensity, walking, and total PA were not significant.

Table 1 also presents values of PT in the two groups of women. In case of PT values for extension, statistically significant differences were found neither in the dominant leg nor in the non-dominant leg. However, in case of difference between PT in non-dominant leg, the value of effect size suggests potentially higher values in the group of women with lower risk of falls.

\section{Discussion}

The main finding of our study, focused on women older than 50, was that the subgroup of women with strength asymmetry between concentric PT of quadriceps of dominant and non-dominant lower leg performed significantly lower volume of vigorous PA than subgroup without strength asymmetry. Considering the fact that it was suggested that strength asymmetry between muscle strength and power of quadriceps could be one of the reasons of falls in elderly (Portegijs, 2008; Skelton et al., 2002), our finding could indicate that participation in vigorous PA is a factor for prevention of muscular imbalances of knee extensors in 
Table 1

Comparison of strength and PA in women being at higher and lower risk of falls associated with bilateral strength asymmetry of knee extensors

\begin{tabular}{|c|c|c|c|c|c|c|}
\hline & \multicolumn{2}{|c|}{ Risk higher $(n=10)$} & \multicolumn{2}{|c|}{ Risk lower $(n=32)$} & \multirow[b]{2}{*}{$p$} & \multirow[b]{2}{*}{$d$} \\
\hline & $M d n$ & $I Q R$ & $M d n$ & $I Q R$ & & \\
\hline \multicolumn{7}{|c|}{ Concentric extension peak torque $(\mathrm{Nm})$} \\
\hline Dominant leg & 89 & 50 & 103.5 & 45 & .27 & 0.3 \\
\hline Non-dominant leg & 82 & 33 & 107.5 & 38 & .07 & 0.6 \\
\hline \multicolumn{7}{|l|}{ Pedometer (steps/day) } \\
\hline Average week day & 8674 & 3330 & 8774 & 4032 & .81 & 0.1 \\
\hline Average work day & 9020 & 4924 & 8806 & 4256 & .75 & 0.1 \\
\hline Average weekend day & 7808 & 5455 & 7215 & 4211 & .58 & 0.2 \\
\hline \multicolumn{7}{|c|}{ IPAQ (METminutes/week) } \\
\hline Vigorous PA & 0 & 0 & 180 & 675 & .04 & 0.6 \\
\hline Moderate PA & 520 & 748 & 660 & 1358 & .47 & 0.2 \\
\hline Walking & 660 & 433 & 1040 & 1299 & .50 & 0.2 \\
\hline Total PA & 1624 & 1771 & 2322 & 1946 & .09 & 0.5 \\
\hline
\end{tabular}

Note. $\mathrm{PA}=$ physical activity, IPAQ $=$ International Physical Activity Questionnaire. $M d n=$ median, $I Q R=$ interquartile range, $p=$ statistical significance (Mann-Whitney $U$ test), $d=$ Cohen's $d$

elderly women. Also Brach, Simonsick, Kritchevsky, Yaffe, and Newman (2004), who observed older men and women (age 70-79 years) and found out that subjects from exercise group (vigorous intensity PA) had better physical function than lifestyle active subjects (daily chores and normal walking), suggested that not only the amount of PA, but also its intensity was important. This suggestion is in agreement with the results of a study which confirmed that regular exercise, resp. PA with higher intensity confers greater benefits than moderate-intensity PA in elderly (Visser et al., 2005).

These findings also correspond to the suggestion that at least loads of $45 \%$ to $50 \%$ of 1 repetition maximum are needed to increase strength in previously untrained individuals (Anderson \& Kearney, 1982; Stone \& Coulter, 1994). Also Ekstrom, Donatelli, and Carp (2007) based on the measurement of muscle activity with EMG suggest that exercises that produced EMG signal amplitude in the muscles on an average greater than $45 \%$ maximal voluntary isometric contraction may provide sufficient stimulus for strength gains in some individuals. Thus, it seems that walking is not sufficient factor which can positively influence strength of knee extensors in elderly (O'Toole, 1997; Visser et al., 2005), although it is the most common PA in this population.

Our results also support the recommendation of World Health Organization (2010) for adults and older adults to participate in muscle-strengthening activities involving major muscle groups on at least 2 days per week.
Taking into account that both volume and intensity of PA in adults decrease with age (Hughes et al., 2001; Pelclová, Vašíčková, Frömel, \& Djordjevic, 2009), we could expect that women from our group with strength asymmetry are in potential higher risk of future falls because of further increase in strength asymmetry (Carabello et al., 2010; Perry et al., 2007) and decrease in strength (Borges, 1989; Keller \& Engelhardt, 2013).

In the present study we did not confirm statistical differences in other observed parameters of PA between the subgroups with and without strength asymmetry of quadriceps of dominant and non-dominant lower limb. However differences in total PA were on the border of statistical significance $(p=.09 ; d=0.5)$. The importance of the volume of PA suggest also results of the study Visser et al. (2005) in elderly population, in which knee extensors muscle strength and also other observed muscle parameter were related to total PA. This finding indicates that also volume of PA in elderly may influence strength of knee extensors of both limbs and thus, perhaps, its asymmetry. However, in this study, compared to our group, older men and women of the age 70-79 year were observed.

Based on the literature review and also results of our study it is evident that aging and PA are related to muscle strength and power and that their development and preservation, including knee extensors and their side to side difference, are important for elderly population for mobility and fall prevention. Therefore we assume that strength asymmetry diagnostics in lower limb muscles may bring information which 
may help to promote quality of life. This suggestion is supported by the results of recent studies (Perry et al., 2007; Semaltianoy et al., 2011). Perry et al. (2007) confirmed in a group of people over 70 years that fallers were more asymmetrical than non-fallers in case of quadriceps. Fallers were also more asymmetrical than non-fallers in power, although in both cases the difference was not statistically significant. Semaltianoy et al. (2011) observed elderly men and women aged 60 year and more and reported significant differences between fallers and non-fallers in isokinetic concentric PT of quadriceps and isometric strength of quadriceps and hamstrings of dominant and nondominant lower limb. Also in the study Skelton et al. (2002), fallers tended to be weaker on muscle strength of the quadriceps and significant differences were also found in case of strength of other muscles to discriminate between fallers and non-fallers in the group of older women (Skelton, 2002). Oppositely, Melzer, Benjuya, and Kaplanski (2004) did not find significant differences between leg muscles strength in elderly fallers and non-fallers.

It has been proven that not only the level of asymmetry is important for falls incidence in elderly population, but also a reduced strength of lower limbs is a contributing factor (Ikezoe, Asakawa, Shima, \& Ichihashi, 2009; Semaltianoy et al., 2011; Skelton et al., 2002). Therefore we compared average PT value of subgroups with lower and higher strength asymmetry with normative values of knee extensors PT at the angular velocity of $180^{\circ} \cdot \mathrm{s}^{-1}$ published by Freedson, Gilliam, Mahoney, Maliszweski, and Kastango (1993). Regardless of the methodological differences between the measurements (in our study strength was measured with gravity compensation, in case of study Freedson et al., gravity compensation was not used) the comparison indicated that in case of both our subgroups average PT value was above average. This fact confirmed also comparison with normative PT of knee extensors at angular velocity of $150^{\circ} \cdot \mathrm{s}^{-1}$ presented by Borges (1989) which showed that the average values of knee extensors PT of observed females were higher than average values for this lower velocity.

The strength of the quadriceps muscle was found to be very important for performing everyday tasks activities (Semaltianoy et al., 2011; Shumway-Cook, Brauer, $\&$ Woollacott, 2000). Nevertheless explosive power of lower limbs is even more strongly related to ability to perform everyday tasks than strength (Skelton et al., 1994). Furthermore results of some studies (Carabello et al., 2010; Skelton et al., 2002) confirmed that the asymmetry of the ability to quickly generate force is more predictive of risk for falls and mobility impairment in elderly women than the muscle strength asymmetry itself. From this point of view, a limitation of the current study is that in our group muscle strength was measured with isokinetic dynamometer. However, isokinetic dynamometry is considered an objective and reliable diagnostic tool which enables the identification of torque through a given range of movement and during varying limb velocities (Baltzopoulos \& Brodie, 1989; Dirnberger, Kösters, \& Müller, 2012). It is also considered to be a safe method of strength assessment in elderly (Perrin, 1993). Another limitation of this study is that only strength of quadriceps at one isokinetic speed was measured.

\section{Conclusions}

The results of our study suggest that strength asymmetry of knee extensors in middle-aged women could be influenced by their participation in vigorous PA. Considering the fact that results of some studies indicate that strength asymmetry of leg muscles including knee extensors is a risk factor for falls, we recommend to take this suggestion into account in habitual PA and training programs in middle-aged population. Based on the results of our study we also suggest that diagnostics of strength asymmetry in lower limb muscles in women over fifty may bring information which can help to avoid the increase in injury risk in later life and may promote quality of life in middle-aged population.

\section{Acknowledgment}

This study was supported by a grant from Czech Science Foundation "Analysis of health-related benefits of walking: Assessment of walking intervention in sedentary adults" (No. 13-32105S).

\section{References}

Anderson, T., \& Kearney, J. T. (1982). Effects of three resistance training programs on muscular strength and absolute and relative endurance. Research Quarterly in Exercise and Sport, 53, 1-7.

Aniansson, A., Hedberg, M., Henning G. B., \& Grimby, G. (1986). Muscle morphology, enzymatic activity, and muscle strength in elderly men: A follow-up study. Muscle Nerve, 9, 585-591.

Baltzopoulos, V., \& Brodie, D. A. (1989). Isokinetic dynamometry: Applications and limitations. Sports Medicine, 8 , 101-116.

Borges, O. (1989) Isometric and isokinetic knee extension and flexion torque in men and women aged 20-70. Scandinavian Journal of Rehabilitation Medicine, 21, 45-53.

Brach, J. S., Simonsick, E. M., Kritchevsky, S., Yaffe, K., \& Newman, A. B. (2004). The association between physical 
function and lifestyle activity and exercise in the health, aging and body composition study. Journal of the American Geriatrics Society, 52, 502-509.

Carabello, R. J., Reid, K. F., Clark, D. J., Philips, E. M., \& Fielding, R. A. (2010). Lower extremity strength and power asymmetry assessment in healthy and mobility-limited populations: Reliability and association with physical functioning. Aging Clinical and Experimental Research, 22, 324-329.

Cohen J. (1988). Statistical power analysis for the behavioral sciences. New York, NY: Lawrence Erlbaum Associates.

Craig, C. L., Marshall, A. L., Sjostrom, M., Bauman, A. E., Booth, M. L., Ainsworth, B. E., ... Oja, P. (2003). International physical activity questionnaire: 12-country reliability and validity. Medicine \& Science in Sports \& Exercise, 35, 1381-1395.

Daly, R. M., Ahlborg, H. G., Ringsberg, K., Gardsell, P., Sernbo, I., \& Karlsson, M. K. (2008). Association between changes in habitual physical activity and changes in bone density, muscle strength, and functional performance in elderly men and women. Physical Activity and Musculoskeletal Health, 56, 2252-2260.

Davies, G. J., Heiderscheit, B., \& Brinks, K. (2000). Test interpretation. In L. E. Brown (Ed.), Isokinetics in Human Performance (pp. 3-24). Champaign, IL: Human Kinetics.

Dirnberger, J., Kösters, A., \& Müller, E. (2012). Concentric and eccentric isokinetic knee extension: A reproducibility study using the IsoMed 2000-dynamometer. Isokinetics and Exercise Science, 20, 31-35.

Ekstrom, R. A., Donatelli, R. A., \& Carp, K. C. (2007). Electromyographic analysis of core trunk, hip, and thigh muscles during 9 rehabilitation exercises. Journal of Orthopaedic and Sports Physical Therapy, 37, 754-762.

Freedson, P. S., Gilliam, T. B., Mahoney, T., Maliszweski, A. F., \& Kastango, K. (1993). Industrial torque levels by age group and gender. Isokinetic and Exercise Science, 3, 34-42.

Frontera, W. R., Hughes, V. A., Fielding, R. A., Fiatarone, M. A., Evans, W. J., \& Roubeno, V. R. (2000). Ageing of skeletal muscle: A 12 year longitudinal study. Journal of Applied Physiology, 88, 1321-1326.

Gauchard, G. C., Tessier, A., Jeandel, C., \& Perrin, P. P. (2003). Improved muscle strength and power in elderly exercising regularly. International Journal of Sports Medicine, 24, 71-74.

Hughes, V. A., Frontera, W. R., Wood, M., Evans, W. J., Dallal, G. E., Roubenoff, R., \& Fiatarone Singh, M. A. (2001). Longitudinal muscle strength changes in older adults: Influence of muscle strength, physical activity, and health. Journals of Gerontology Series A: Biological Sciences and Medical Sciences, 56, 209-217.

Hunter, S. K., Thompson, M. W., \& Adams, R. (2000). Relationship among age-associated strength changes and physical activity level, limb dominance, and muscle group in women. Journals of Gerontology Series A: Biological Sciences and Medical Sciences, 55, 264-273.

Ikezoe, T., Asakawa, Y., Shima, H., \& Ichihashi, N. (2009). Physical function screening of institutionalized elderly women to predict their risk of falling. Japanese Journal of Physical Fitness and Sport Medicine, 58, 489-498.

IPAQ group. (2014a). Cultural adaptation. Retrieved from https://sites.google.com/site/theipaq/cultural-adaptation
IPAQ group. (2014b). IPAQ scoring protocol. Retrieved from https://sites.google.com/site/theipaq/scoring-protocol

Kapandji, I. A. (2002). The physiology of the joints. Volume two, lower limb. Edinburgh: Churchill Livingstone.

Keller, K., \& Engelhardt, M. (2013). Strength and muscle mass loss with aging process: Age and strength loss. Muscles, Ligaments and Tendons Journal, 3, 346-350.

King, A. C., Rejeski, W. J., \& Buchner, D. M. (1998). Physical activity interventions targeting older adults: A critical review and recommendations. American Journal of Preventive Medicine, 15, 316-333.

Lanshammar, K., \& Ribom, E. L. (2011). Differences in muscle strength in dominant and non-dominant leg in females aged 20-39 years: A population-based study. Physical Therapy in Sport, 12, 76-79.

LaRoche, D. P., Cook, S. B., \& Mackala, K. (2012). Strength asymmetry increases gait asymmetry and variability in older women. Medicine \& Science in Sports \& Exercise, 44, 2172-2181.

Lindle, R. S., Metter, E. J., Lynch, N. A., Fleg, J. L., Fozard, J. L., Tobin, J., ... Hurley, B. F. (1997). Age and gender comparisons of muscle strength in 654 women and men aged 20-93. Journal of Applied Physiology, 83, 1581-1587.

Macaluso, A., \& De Vito, G. (2004). Muscle strength power and adaptations to resistance training in older people. European Journal of Applied Physiology, 91, 450-472.

Melzer, I., Benjuya, N., \& Kaplanski, J. (2004). Postural stability in the elderly: A comparison between fallers and non-fallers. Age and Ageing, 33, 602-607.

Ochi, M., Tabara, Y., Kido, T., Uetani, E., Ochi, N., Igase, M., ... Kohara, K. (2010). Quadriceps sarcopenia and visceral obesity are risk factors for postural instability in the middle-aged to elderly population. Geriatrics and Gerontology International, 10, 233-243.

O'Toole, M. L. (1997). Do older individuals need more than usual physical activities to maintain muscle strength and function? Journal of the American Geriatrics Society, 45, 1534-1535.

Pelclová, J., Vašičková, J., Frömel, K., \& Djordjevic, I. (2009). Leisure time, occupational, domestic, and commuting physical activity of inhabitants of the Czech Republic aged 55-69: Influence of socio-demographic and environmental factors. Acta Universitatis Palackianae Olomucensis. Gymnica, 39(3), 13-20.

Perry, M. C., Carville, S. F., Smith, I. C., Rutherford, O. M., \& Newham, D. J. (2007). Strength, power output and symmetry of leg muscles: Effect of age and history of falling. European Journal of Applied Physiology, 100, 553-561.

Perrin D. (1993). Isokinetic exercise and assessment. Champaign, IL: Human Kinetic.

Portegijs, E. (2008). Assymetrical lower-limb muscle strength deficit in older people. Jÿväskylä: University of Jÿväskylä.

Sadeghi, H., Allard, P., Prince, F., \& Labelle, H. (2000). Symmetry and limb dominance in able-bodied gait: A review. Gait and Posture, 12, 34-45.

Semaltianoy, E., Kabitsis, C., Harahousou, Y., \& Lazou, V. (2011). Investigation of fall prediction factors in elderly population using isokinetic assessment of the knee joint. Biology of Exercise, 7(2), 49-62.

Shumway-Cook, A., Brauer, S., \& Woollacott, M. (2000). Predicting the probability for falls in community-dwelling 
older adults using the Timed Up \& Go Test. Physical Therapy, 80, 896-903.

Skelton, D. A., Greig, C. A., Davies, J. M., \& Young, A. (1994). Strength, power and related functional ability of healthy people aged 65-89 years. Age and Ageing, 23, 371-377.

Skelton, D. A., Kennedy, J., \& Rutherford, O. M. (2002). Explosive power and asymmetry in leg muscle function in frequent fallers and non-fallers aged over 65. Age and Ageing, 31, 119-125.

Stokes, J., \& Linsay, J. (1996). Major causes of death and hospitalization in Canadian seniors. Chronic Diseases in Canada, 17, 63-73.

Stoll, T., Hubert, E., Seifert, B., Michel, B. A., \& Stucki, G. (2000). Maximal isometric muscle strength: Normative values and gender-specific relation to age. Clinical Rheumatology, 19, 105-113.

Stone, W. J., \& Coulter, S. P. (1994). Strength/endurance effects from three resistance training protocols with women. Journal of Strength and Conditioning Research, 8, 231-234.

Suetta, C., Aagaard, P., Magnusson, S. P., Andersen, L. L., Sipila, S., Rosted, A., ... Kjaer, M. (2007). Muscle size, neuromuscular activation, and rapid force characteristics in elderly men and women: Effects of unilateral long-term disuse due to hip-osteoarthrosis. Journal of Applied Physiology, 102, 942-948.
Tudor-Locke, C., Bassett, D. R., Shipe, M. F., \& McClain, J. J. (2011). Pedometry methods for assessing free-living adults. Journal of Physical Activity and Health, 8, 445-453.

Visser, M., Simonsick, E. M., Colbert, L. H., Brach, J., Rubin, S. M., Kritchevsky, S. B., ... Harris, T. B. (2005). Type and intensity of activity and risk of mobility limitation: The mediating role of muscle parameters. Journal of the American Geriatrics Society, 53, 762-770.

Véle, F. (2006). Kineziologie: přehled klinické kineziologie a patokineziologie pro diagnostiku a terapii poruch pohybové soustavy [Overview of clinical kinesiology and pathokinesiology for the diagnostics and treatment of musculoskeletal disorders] (2nd ed.). Praha: Triton.

Von Haehling, S., Morley, J. E., \& Anker, S. D. (2010). An overview of sarcopenia: Facts and numbers on prevalence and clinical impact. Journal of Cachexia, Sarcopenia and Muscle, 1, 129-133.

World Health Organization. (2010). Global recommendations on physical activity for health. Retrieved from http://whqlibdoc.who.int/publications/2010/9789241599979_eng.pdf Yogev, G., Plotnik, M., Peretz, C., Giladi, N., \& Hausdorff, J. M. (2007). Gait asymmetry in patients with Parkinson's disease and elderly fallers: When does the bilateral coordination of gait require attention? Experimental Brain Research, 177, 336-346. 